\title{
LIX. Demonstration of some useful theorems in the geometry of coordinates
}

\section{William Rutherford Esq. F.R.A.S.}

To cite this article: William Rutherford Esq. F.R.A.S. (1843) LIX. Demonstration of some useful theorems in the geometry of coordinates, Philosophical Magazine Series 3, 22:146, 353-358, DOI: $10.1080 / 14786444308636395$

To link to this article: http://dx.doi.org/10.1080/14786444308636395

册 Published online: 01 Jun 2009.

Submit your article to this journal $[\pi$

Џ Article views: 2

Q View related articles 
means a common word among mathematicians; it was a technical term of judicial astrology, and would probably be avoided by the geometer: Torporley was an astrologer, as appears from the opening of his work. Trias and ternio would suggest themselves first (trinitas being excluded for an obvious reason). On this word probably the question will turn: if it should be found that no mathematicians of the period use the word triplicitas except Torporley and Napier, it will be difficult to avoid presuming that the latter must have seen the work of the former.

I remain, Gentlemen, Yours faithfully,

\section{University College, March 13, 1843. \\ A. De Morgan.}

LIX. Demonstration of some useful Theorems in the Geometry of Coordinates. By WiLLIaM Rutherford, Esq., F.R.A.S., Royal Military Academy*.

THEOREM I.-If the equations of two straight lines be

$$
\frac{x}{\alpha}+\frac{y}{\beta}=1 \text { and } \frac{x}{\alpha_{1}}+\frac{y}{\beta_{1}}=1,
$$

then will the sum of these equations, viz.

$$
\left\{\frac{1}{\alpha}+\frac{1}{\alpha_{1}}\right\} x+\left\{\frac{1}{\beta}+\frac{1}{\beta_{1}}\right\} y=2,
$$

be the equation of the straight line passing through the point of intersection of these lines, and the point of intersection of the diagonals of the quadrilateral formed by the intersection of the given lines with the axes of coordinates.

Let $\mathrm{OX}, \mathrm{OY}$ be the axes of coordinates having any angle of ordination, and let $P, Q$, $R, S$ be any four points in these coordinate axes; then if $\mathrm{OP}=\alpha, \mathrm{OQ}=\alpha_{1}, \mathrm{OS}=\beta$, and $\mathrm{OR}=\beta_{1}$, the equations of the lines $\mathrm{S} P$ and $R Q$, drawn through the points $S, P$ and $\mathrm{R}, \mathrm{Q}$ are respectively

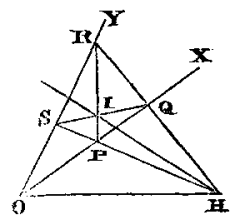

$$
\frac{x}{\alpha}+\frac{y}{\beta}=1 \text { and } \frac{x}{\alpha_{1}}+\frac{y}{\beta_{1}}=1 . . .
$$

Now these lines must either be parallel, or they will meet if produced. Let them meet when produced in $\mathrm{H}$, and let I be the point of intersection of the diagonals $S Q$ and $R P$ of the quadrilateral PQR S. Join $\mathrm{OH}$, and through $\mathrm{H}$ and $\mathrm{I}$

* Communicated by the Author. 
draw the straight line HI. Then the equations of the diagonals $\mathbf{P} \mathbf{R}$ and $\mathbf{S} \mathbf{Q}$ are respectively

$$
\frac{x}{\alpha}+\frac{y}{\beta_{1}}=1 \text { and } \frac{x}{\alpha_{1}}+\frac{y}{\beta}=1 ; . . \text {. }
$$

and since the point $H$ is common to both the lines $S P$ and $\mathrm{RQ}$; and the point I common to both the diagonals $\mathbf{S Q}$ and $\mathrm{P} R$; therefore the sum of the equations in (1.), viz.

$$
\left\{\frac{1}{\alpha}+\frac{1}{\alpha_{1}}\right\} x+\left\{\frac{1}{\beta}+\frac{1}{\beta_{1}}\right\} y=2, .
$$

is evidently the equation of a line passing through $H$ the point of intersection of the lines denoted by the equations (1.); but equation (3.) is also the sum of the equations (2.), and therefore equation (3.) is likewise the equation of a line passing through $I$ the point of intersection of the diagonals of the quadrilateral $\mathrm{PQRS}$; hence the truth of the theorem is established.

If the lines $\mathbf{S} \mathbf{P}$ and $\mathbf{R} \mathbf{Q}$ are parallel, then the triangles OP S, OQR will be equiangular, and therefore we have the relation

$$
\frac{\alpha}{\alpha_{1}}=\frac{\beta}{\beta_{1}}, \text { or } \beta_{1}=\frac{\alpha_{1}}{\alpha} \beta
$$

and this value being substituted for $\beta_{1}$ in equation (3.), gives

$$
\frac{\alpha_{1}+\alpha}{2 \alpha_{1}}\left\{\frac{x}{\alpha}+\frac{y}{\beta}\right\}=1 \text {, or } \frac{x}{\alpha}+\frac{y}{\beta}=\frac{2 \alpha_{1}}{\alpha_{1}+\alpha} \text {. }
$$

which is the equation of the straight line parallel to the given line

$$
\frac{x}{\alpha}+\frac{y}{\beta}=1,
$$

and passing through I the point of intersection of the diagonals of the trapezoid PQR S.

Cor.-Hence it is obvious that the difference of the equations in (1.), viz.

$$
\left\{\frac{1}{\alpha}-\frac{1}{\alpha_{1}}\right\} x+\left\{\frac{1}{\beta}-\frac{1}{\beta_{1}}\right\} y=0, \ldots
$$

is the equation of the straight line $\mathrm{OH}$ passing through the origin of coordinates, and the point of intersection of the two lines $\mathbf{S} \mathbf{P}$ and $R \mathbf{Q}$. If these lines are parallel, then we have

$$
\beta_{1}=\frac{\alpha_{1}}{\alpha} \beta
$$

and by substituting this value of $\beta_{1}$ in equation (5.), we have

$$
\frac{\alpha_{1}-\alpha}{\alpha_{1}}\left\{\begin{array}{l}
x \\
\alpha
\end{array}+\frac{y}{\beta}\right\}=0 \text {, or } \frac{x}{\alpha}+\frac{y}{\beta}=0, .
$$


which is the equation of the line passing through the origin, and parallel to the line whose equation is

$$
\frac{x}{\alpha}+\frac{y}{\beta}=1 \text {. }
$$

Theorem II.-If the equations of two planes be represented by

then will the sum of these equations, viz.

$$
\frac{x}{\alpha}+\frac{y}{\beta}+\frac{z}{\gamma}=1 \text { and } \frac{x}{\alpha_{1}}+\frac{y}{\beta_{1}}+\frac{z}{\gamma_{1}}=1,
$$

$$
\left\{\frac{1}{\alpha}+\frac{1}{\alpha_{1}}\right\} x+\left\{\frac{1}{\beta}+\frac{1}{\beta_{1}}\right\} y+\left\{\frac{1}{\gamma}+\frac{1}{\gamma_{1}}\right\} z=2,
$$

be the equation of the plane passing through the line of intersection of these planes, and through the three points of intersection of the diagonals of the three quadrilaterals formed by the intersection of the given planes with the axes of coordinates.

Let $\mathrm{OX}, \mathrm{OY}, \mathrm{OZ}$ be the oblique axes of coordinates, and let $P, Q, R$ be any three points in the axes of $x, y, z$ respectively, and $P^{\prime}, Q^{\prime}, R^{\prime}$ any other three points in the same axes. Draw the traces $\mathrm{P} \mathbf{Q}, \mathbf{Q} \mathbf{R}, \mathbf{R} \mathrm{P}, \mathbf{P}^{\prime} \mathbf{Q}^{\prime}, \mathbf{Q}^{\prime} \mathbf{R}^{\prime}, \mathbf{R}^{\prime} \mathrm{P}^{\prime}$ forming with the coordinate axes the three quadrilaterals $\mathbf{P} \mathbf{Q Q}^{\prime} \mathbf{P}^{\prime}$, $\mathrm{Q} R \mathrm{R}^{\prime} \mathrm{Q}^{\prime}, \mathrm{PR} \mathrm{R}^{\prime} \mathrm{P}^{\prime}$, and let $\mathrm{I}, \mathrm{H}$, $G$ be the points of intersection of the diagonals of these quadrilaterals respectively. Then if we put \begin{tabular}{l|l|l}
$\mathrm{OP}=\alpha$ & $\mathrm{OQ}=\beta$ & $\mathrm{OR}=\gamma$ \\
$\mathrm{OP}=\alpha_{1}$ & $\mathrm{OQ} \mathrm{Q}^{\prime}=\beta_{3}$ & $\mathrm{OR}=\gamma_{1}^{\prime}$,
\end{tabular}

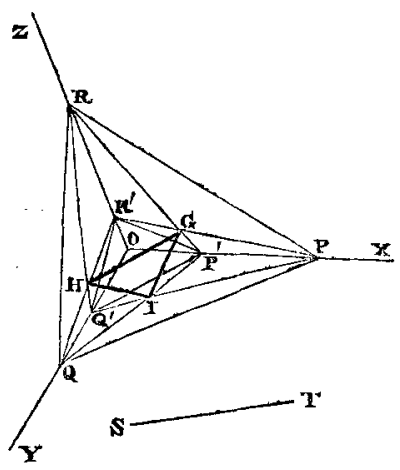
we shall have the equations of the several planes as below.

$$
\begin{aligned}
& \text { (P Q R) . } \frac{x}{\alpha}+\frac{y}{\beta}+\frac{z}{\gamma}=1 \ldots . \\
& \text { ( } \left.\mathrm{P}^{\prime} \mathrm{Q} \mathrm{R}\right) \quad \cdot \frac{x}{\alpha_{1}}+\frac{y}{\beta}+\frac{z}{\gamma}=1 \ldots . . \\
& \left(\mathrm{P} \mathrm{Q} \mathrm{Q}^{\prime} \mathrm{R}\right) \quad \cdot \frac{x}{\alpha}+\frac{y}{\beta_{1}}+\frac{z}{\gamma}=1 \quad . . . \\
& \left(\mathrm{P} \mathrm{Q} \mathrm{R}^{\prime}\right) \cdot \cdot \frac{x}{\alpha}+\frac{y}{\beta}+\frac{z}{\gamma_{1}}=1 \quad . . . \\
& \left(\mathrm{P}^{\prime} \mathbf{Q}^{\prime} \mathbf{R}^{\prime}\right) \cdot \frac{x}{\alpha_{1}}+\frac{y}{\beta_{1}}+\frac{z}{\gamma_{1}}=1 \quad \cdot \cdot \text {. } \\
& \left(\mathrm{PQ}^{\prime} \mathrm{R}^{\prime}\right) \cdot \frac{x}{\alpha}+\frac{y}{\beta_{1}}+\frac{z}{\gamma_{1}}=1 \text {... . }
\end{aligned}
$$




$$
\begin{aligned}
& \left(\mathrm{P}^{\prime} \mathrm{Q} \mathrm{R}^{\prime}\right) \cdot \cdot \frac{x}{\alpha_{1}}+\frac{y}{\beta}+\frac{z}{\gamma_{1}}=1 \quad . \quad \therefore . \\
& \left(\mathrm{P}^{\prime} \mathrm{Q}^{\prime} \mathrm{R}\right) \quad \cdot \frac{x}{\alpha_{1}}+\frac{y}{\beta_{1}}+\frac{z}{\gamma}=1 \quad . \quad . .
\end{aligned}
$$

Now if we take the sum of the equations (1.) and (5.), (2.) and (6.), (3.) and (7.), (4.) and (8.), we shall, in each case, have the same resulting equation, viz.

$$
\left\{\frac{1}{\alpha}+\frac{1}{\alpha_{1}}\right\} x+\left\{\frac{1}{\beta}+\frac{1}{\beta_{1}}\right\} y+\left\{\frac{1}{\gamma}+\frac{1}{\gamma_{1}}\right\} z=2 .
$$

Let S T be the line of intersection of the two planes in (1.) and (5.); that is of the planes $P Q R$ and $P^{\prime} Q^{\prime} R^{\prime}$; then the line $S T$ being common to the planes $P Q R$ and $P^{\prime} Q^{\prime} R^{\prime}$, and since the point $I$ is common to the planes $P^{\prime} Q R$ and $P Q^{\prime} R^{\prime}$, the point $H$ to the planes $P Q^{\prime} R$ and $P^{\prime} Q R^{\prime}$, and the point $G$ to the planes $P Q R^{\prime}$ and $P^{\prime} Q^{\prime} R$; therefore it is obvious that equation (9.), which is the sum of the equations of these planes, taken two and two, is the equation of the plane which passes through the line $S T$ and through the three points $\mathrm{G}, \mathrm{H}, \mathrm{I}$ the points of intersection of the diagonals of the quadrilaterals formed by the two planes $P Q R$ and $P^{\prime} Q^{\prime} R^{\prime}$ with the axes of coordinates.

Cor.-If the equations of two planes be denoted by

$$
\frac{x}{\alpha}+\frac{y}{\beta}+\frac{z}{\gamma}=1 \text { and } \frac{x}{\alpha_{1}}+\frac{y}{\beta_{1}}+\frac{z}{\gamma_{1}}=1,
$$

then will the difference of these equations, viz.

$$
\left\{\frac{1}{\alpha}-\frac{1}{\alpha_{1}}\right\} x+\left\{\frac{1}{\beta}-\frac{1}{\beta_{1}}\right\} y+\left\{\frac{1}{\gamma}-\frac{1}{\gamma_{1}}\right\} z=0,
$$

be the equation of the plane which passes through the line of intersection of these planes, and the origin of coordinates.

The principles developed in these theorems and corollaries are very effective in analytical inquiries, and in order to point out their application I shall add the following demonstration of a well-known theorem.

Theorem.- If straight lines A Q, B R, C S be drawn from the angles $\mathrm{A}, \mathrm{B}, \mathrm{C}$ of a triangle through any point $\mathrm{P}$ to meet the opposite sides in $Q, R, S$; and if $\mathrm{QR}, \mathrm{QS}, \mathrm{R} \mathrm{S}$ be drawn to meet the sides of the triangle in $G, H, I$; then will the points $G, H, I$ be in the same straight line.

Take $\mathrm{H} \mathrm{C,} \mathrm{HQ}$ for the axes of $x$ and $y$ respectively, and put $\mathrm{HA}=\alpha_{1}$, $\mathrm{HR}=\alpha_{2}, \mathrm{HC}=\alpha_{3}, \mathrm{HS}=\beta_{1}$, and $\mathrm{HQ}$
$=\beta_{2} ;$ then we have the equations of the several lines as below.

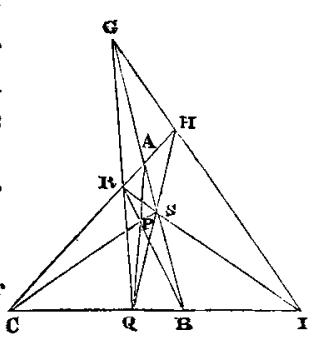



(A B) $\ldots \frac{x}{\alpha_{1}}+\frac{y}{\beta_{1}}=1 \ldots$ (1.)
(R S) $\ldots \frac{x}{\alpha_{2}}+\frac{y}{\beta_{1}}=1 \ldots$
$(\mathrm{GQ}) \ldots \frac{x}{\alpha_{2}}+\frac{y}{\beta_{2}}=1 \ldots$
(AQ) $\ldots \frac{x}{\alpha_{1}}+\frac{y}{\beta_{2}}=1 \ldots$
(B C) $\ldots \frac{x}{\alpha_{3}}+\frac{y}{\beta_{2}}=1 \ldots$
(C S) $\ldots \frac{x}{\alpha_{3}}+\frac{y}{\beta_{1}}=1 \ldots$

Now (Theorem I., Cor.) if we subtract (4.) and (1.) from (3.) and (2.) respectively, we shall have the equations of $H I$ and $H \mathrm{G}$, viz.-

$$
\begin{aligned}
& \text { (H I) } \ldots\left\{\frac{1}{\alpha_{3}}-\frac{1}{\alpha_{2}}\right\} x+\left\{\frac{1}{\beta_{2}}-\frac{1}{\beta_{1}}\right\} y=0 \ldots \\
& \text { (H G) .. }\left\{\frac{1}{\alpha_{2}}-\frac{1}{\alpha_{3}}\right\} x+\left\{\frac{1}{\beta_{2}}-\frac{1}{\beta_{1}}\right\} y=0 \ldots
\end{aligned}
$$

These equations will be identical if it can be shown that the coefficients of $x$ are equal, and to effect this, the condition that the lines A Q, B R, C S all pass through the same point $P$ must be employed. Hence if we add the equations of $\mathbf{C} \mathbf{Q}$ and $A S$, viz. (3.) and (1.), we shall have the equation of $B P$ (Theorem I.), viz.

$$
\left\{\frac{1}{\alpha_{1}}+\frac{1}{\alpha_{3}}\right\} x+\left\{\frac{1}{\beta_{1}}+\frac{1}{\beta_{2}}\right\} y=2 . . .
$$

To find where this line cuts the axis of $x$, make $y=0$, and we get

$$
x=\frac{2}{\frac{1}{\alpha_{1}}+\frac{1}{\alpha_{3}}} ;
$$

and this must evidently be the value of $H R$, because $B$ P R is a straight line by hypothesis; but $\mathrm{H} \mathrm{R}=\boldsymbol{x}_{2}$, and therefore we must have

$$
\begin{aligned}
& \frac{2}{\frac{1}{\alpha_{1}}+\frac{1}{\alpha_{3}}}=\alpha_{2}, \text { or } \frac{2}{\alpha_{2}}=\frac{1}{\alpha_{1}}+\frac{1}{\alpha_{3}}, \\
\therefore \quad & \frac{1}{\alpha_{2}}-\frac{1}{\alpha_{1}}=\frac{1}{\alpha_{3}}-\frac{1}{\alpha_{2}}, \text { by transposition; }
\end{aligned}
$$

and consequently equations (7.) and (8.) are identical, and therefore the points $\mathrm{G}, \mathrm{H}, \mathrm{I}$ range in the same straight line.

The form of the equation of a straight line used in these inquiries is well adapted to the demonstration of a class of theorems concerning the intersections of straight lines, of which the preceding example is an instance, and a variety of others will be found in the forthcoming second volume of Hutton's Course, by my talented colleague Mr. Davies, 


\section{Mr. A. Cayley's Remarks on the Rev. B. Bronwin's}

where the principle developed in the first of these Theorems is frequently applied with much simplicity, elegance ard advantage. I am not without a hope that the publication of that volume will materially contribute to the improvement of the taste of the young geometer, not only from the great number of original and well-chosen discussions which are introduced into it, but also from the many beautiful investigations with which it is enriched.

I have also to add that my attention was called to the principle of combining the equations of straight lines as here employed by my colleague Mr. Fenwick, and that some further inquiries of this kind will appear in a tract which I am, in conjunction with that gentleman, about to publish, and which may possibly be succeeded by others of a similar nature.

LX. Remarks on the Rev. B.Bronwin's paper on M. Jacobi's Theory of Elliptic Functions. By A. CaYley, Esq., B.A., F.C.P.S., Fellow of Trinity College, Cambridge.

To the Editors of the Philosophical Magazine and Journal.

GenTLemen,

A LLOW me to insert in your Magazine a few remarks on a paper "On M. Jacobi's Theory of Elliptic Functions," which appeared in your last Number, in which the author, Mr. Bronwin, attempts to show that some of M. Jacobi's formulæ are erroneous. As far as I can understand his argument, he wishes to deduce from the equation

$$
\mathrm{s} a \frac{u}{M}=\mathrm{Cs} a u \mathrm{~s} a(u+2 \omega) \ldots \mathrm{s} a(u+2(n-1) \omega)
$$

(numbered (3.) in the paper referred to) the conclusion that $\mathbf{C}$ is necessarily, in all cases in which the formula exists at all, given by the equation

$$
\frac{1}{\mathrm{C}}= \pm \mathrm{s} a \omega \mathrm{s} a 3 \omega \ldots \ldots \ldots \mathrm{s} a(2 n-1) \omega .
$$

Omitting for the present his remarks upon the form $\omega=\frac{2 r \mathrm{~K}+2 r^{\prime} \mathrm{K}^{\prime} \sqrt{-1}}{n}$, for the three remaining forms of $\omega$,

$$
\text { viz. } \begin{aligned}
\omega & =\frac{\overline{2 r+1} \mathrm{~K}+2 r^{\prime} \mathrm{K}^{\prime} \sqrt{-1}}{n}, \\
\omega & =\frac{2 r \mathrm{~K}+\overline{2 r^{\prime}+1} \mathrm{~K}^{\prime} \sqrt{-1}}{n}, \\
\omega & =\frac{\overline{2 r+1} \mathrm{~K}+\overline{2 r^{\prime}+1} \mathrm{~K}^{\prime} \sqrt{-1}}{n},
\end{aligned}
$$

